\section{Effectiveness of limited airway ultrasound education for medical students: a pilot study}

\author{
Seunghun Park, Sanghun Lee, Han Ho Do, Jae Seong Kim, Jun Seok Seo \\ Department of Emergency Medicine, Dongguk University Ilsan Hospital, Dongguk University School of \\ Medicine, Goyang, Korea
}

Objective The point-of-care ultrasound of the airway (POCUS-A) is a useful examination method but there are currently no educational programs for medical students regarding it. We designed a POCUS-A training curriculum for medical students to improve three cognitive and psychomotor learning domains: knowledge of POCUS-A, image acquisition, and image interpretation.

Methods Two hours of training were provided to 52 medical students in their emergency medicine (EM) rotation. Students were evaluated for cognitive and psychomotor skills before and immediately after the training. The validity measures were established with the help of six specialists and eight EM residents. A survey was administered following the curriculum.

Results Cognitive skill significantly improved after the training (38.7 \pm 12.4 vs. $91.2 \pm 7.7)$ and there was no significant difference between medical students and EM residents in posttest scores ( $91.2 \pm 7.7$ vs. $90.8 \pm 4.6)$. The success rate of overall POCUS-A performance was $95.8 \%$. The students were confident to perform POCUS-A on an actual patient and strongly agreed to incorporate POCUS-A training in their medical school curriculum.

Conclusion Cognitive and psychomotor skills of POCUS-A among medical students can be improved via a limited curriculum on EM rotation.

Keywords Ultrasonography; Airway management; Education, medical

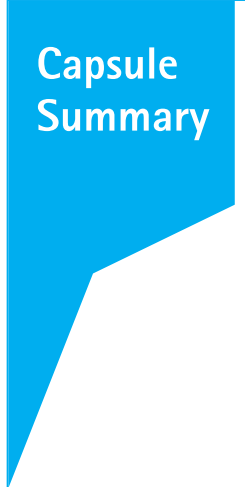

What is already known

Point-of-care ultrasound is increasing because it is convenient, fast, and noninvasive and aids in decision making and the performance of other procedures. Many medical schools have introduced point-of-care ultrasound into the medical curriculum at the undergraduate level.

What is new in the current study

This study shows that simple airway ultrasound education can improve the knowledge and image acquisition skills and interpretation ability of medical students.
eISSN: 2383-4625

Received: 19 July 2018

Revised: 8 September 2018

Accepted: 29 September 2018

Correspondence to: Han Ho Do Department of Emergency Medicine, Dongguk University Ilsan Hospital, 27 Dongguk-ro, Ilsandong-gu, Goyang 10326, Korea

E-mail:erdo@dongguk.edu ORCID

http://orcid.org/0000-0001-6950-9137

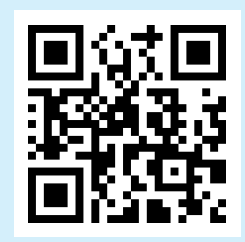

How to cite this article:

Park S, Lee S, Do HH, Kim JS, Seo JS. Effectiveness of limited airway ultrasound education for medical students: a pilot study. Clin Exp Emerg Med 2019;6(3):257263.

This is an Open Access article distributed under the terms of the Creative Commons Attribution Non-Commercial License (http:// creativecommons.org/licenses/by-nc/4.0/). 


\section{INTRODUCTION}

Ultrasonography is noninvasive and allows rapid examination without patient relocation. Therefore, the use of point-of-care ultrasound (POCUS), which is performed at the bedside by the clinician to determine treatment goals or assist with procedures, has increased. With increasing importance of ultrasonography, many medical schools have introduced POCUS into the undergraduate medical curriculum. ${ }^{1-3}$

POCUS of the airway (POCUS-A) provides a rapid assessment of the airway anatomy and facilitates critical airway management through a highly reliable evaluation of the subglottic airway. ${ }^{4}$ POCUS-A enables real-time confirmation of endotracheal tube location and determination of the anatomical position of the cricothyroid membrane (CTM) in cricothyroidotomy. As POCUS-A provides important information quickly and effectively, it is particularly useful in the operating room, intensive care unit, and the emergency department (ED) due to the frequency of airway management requirements. ${ }^{4-6}$

In this pilot study, we sought to test our hypothesis that brief POCUS-A education during ED rotation could improve the cognitive and psychomotor skills regarding its use among medical students.

\section{METHODS}

\section{Study design}

This prospective study was conducted from March to June 2016 at the Dongguk University College of Medicine in South Korea. The study involved 52 medical college students in their clerkship rotation on emergency medicine (EM) during their fourth year in the medical school. This study was performed in accordance with the Declaration of Helsinki. This human study was approved by institutional review board of Dongguk University Ilsan Hospital (DUIH 2016-43). All adult participants provided written informed consent to participate in this study.

The aims of the POCUS-A lesson were to train students to evaluate airway anatomy including the CTM and distinguish between endotracheal and esophageal intubation. To evaluate the effectiveness of the program, students completed a written examination, an ultrasound performance test, and post-training questionnaires (Table 1).

\section{Validity}

In order to confirm the content validity, six specialists (three intensivists and three emergency physicians) experienced in POCUSA assessed the contents of the education and examined the compatibility of the items on a 4-point modified Likert scale $(1=$ not
Table 1. Development of point-of-care ultrasound of the airway educational program

\begin{tabular}{|c|c|c|}
\hline $\begin{array}{l}\text { Developing the edu- } \\
\text { cational program }\end{array}$ & Contents & Methods \\
\hline Design & Validity & $\begin{array}{l}\text { Content validity using CVI } \\
\text { Face validity } \\
\text { Construct validity }\end{array}$ \\
\hline Pretest & Theoretical, practical & PowerPoint slide \\
\hline Education & $\begin{array}{l}\text { Theoretical } \\
\text { Practical }\end{array}$ & $\begin{array}{l}\text { PowerPoint slide } \\
\text { Healthy standard patient }\end{array}$ \\
\hline Posttest & Theoretical, practical & PowerPoint slide \\
\hline Survey & Self-assess & Questionnaire with Likert scale \\
\hline
\end{tabular}

$\mathrm{CVI}$, contents validity index.

relevant, $2=$ somewhat relevant, $3=$ quite relevant, and $4=$ highly relevant). The content validity index (CVI) was calculated based on the number of experts who scored greater than 3 on the conformity scale. The educational content was selected when the $\mathrm{CVI}$ score was 0.83 (5/6) or higher. In order to assess the face validity, eight EM residents at various years of training were selected. They completed the pretest, posttest, and post-training survey; then their feedback was reflected in the revisions to improve the clarity of the items. Construct validity was confirmed by applying the post-training test results of the students to the results of eight EM residents.

\section{POCUS-A education}

We designed a curriculum based on the tracheal rapid ultrasound examination method proposed by Chou et al. ${ }^{7}$ to identify endotracheal tube placement and ultrasound guide by Mallin et al. ${ }^{8}$ to identify CTM. POCUS-A education consisted of an hour of theoretical education and practical training each.

Theoretical education included the basic principles and operation of ultrasonography, airway anatomical structure, and ultrasonographic findings during endotracheal and esophageal intubation. Ultrasonographic findings were described using still images and video clips. Theoretical training was performed using previously recorded PowerPoint slides and practical training was conducted with a standard healthy patient. The subglottic airway structure of the standard patient lying in a supine position was assessed using ultrasonography and the movement of the esophagus was determined by asking patients to swallow saliva while students concurrently observed both the trachea and esophagus. Ultrasonography was performed in the following order from the level of thyroid cartilage to the sternal notch in the anterior part of the neck (Fig. 1). 1) Transverse scan of thyroid cartilage, 2) transverse scan of cricoid cartilage, 3 ) transverse scan of trachea and esophagus (below cricoid cartilage level), 4) transverse scan of 


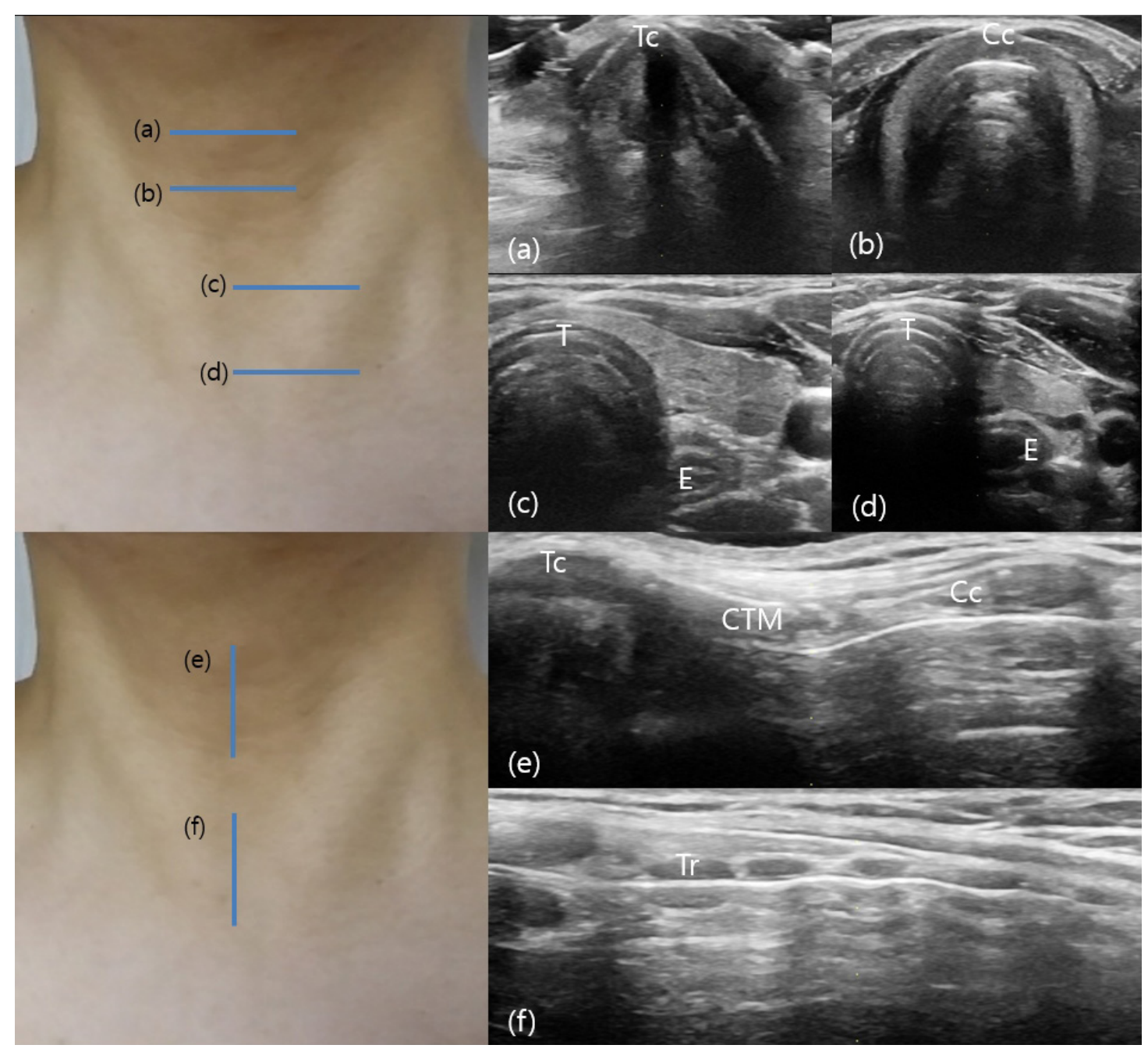

Fig. 1. Location of probe for the point-of-care ultrasound of the airway. (a) Transverse scan of thyroid cartilage, (b) transverse scan of cricoid cartilage, (c) transverse scan of trachea and esophagus (below cricoid cartilage), (d) transverse scan of trachea and esophagus (above sternal notch), (e) longitudinal scan of cricothyroid membrane, and (f) longitudinal scan of tracheal cartilage. Tc, thyroid cartilage; $\mathrm{Cc}_{1}$ cricoid cartilage; $\mathrm{T}_{\text {, trachea; }}$, esophagus; CTM, cricothyroid membrane; $T r$, tracheal ring.

trachea and esophagus (above sternal notch level), 5) longitudinal scan of cricothyroid membrane, and 6) longitudinal scan of tracheal cartilage.

The curriculum supervisor was an EM specialist with over 10 years of experience in emergency ultrasound and POCUS education. Ultrasonography was performed using a SONOACE X8 (Samsung Medison, Seongnam, Korea) and a 5-12 MHz linear probe (L512EC, Samsung Medison) was used as the transducer.

\section{Evaluation of the effectiveness of education}

Tests were conducted before and after the training to assess cognitive skills. A total of 15 questions were administered including three about the principles of ultrasound, six identifying the airway and surrounding anatomical structures with ultrasound im- ages, and six identifying whether the endotracheal tube was located in the airway through ultrasound examination. The psychomotor skills were evaluated as success or failure, based on proficiency in image acquisition and identification (Supplementary Materials). The ultrasound performance and quality of the acquired image were evaluated by a 4th-year EM resident and the appropriateness of the acquired image was rechecked by the EM staff who was the supervisor of education. After the training, we evaluated students' degree of satisfaction with the education, their fluency in use of POCUS-A, need for airway ultrasound education in medical schools, and their confidence in the possible use of airway ultrasound in real patients. The questionnaire was answered using a 5 -point Likert scale $\left(1=\right.$ very low, $2=\operatorname{low}_{1} 3=$ moderate, $4=$ high, $5=$ very high). 


\section{Statistical analysis}

Categorical variables were expressed as frequency and percentage. Data were expressed as mean and standard deviation when they showed normal distribution, and median and quartiles when they failed to show normality. An independent t-test was conducted to determine the construct validity. The students' performance before and after the training was compared using the paired t-test. Statistical analysis was performed using PASW Statistics ver.18 (SPSS Inc., Chicago, IL, USA) and a P-value of less than 0.05 was considered statistically significant.

\section{RESULTS}

\section{General characteristics}

A total of 52 students including 36 males and 16 females completed the curriculum. All students completed pre- and post-training written tests, ultrasound performance tests, and posttest surveys. The average age of the students was $27.6 \pm 3.2$ years. Thirtysix students had previous experience of theoretical education of general ultrasound, and five of them had experience in hands-on ultrasound training. However, none of the students had prior knowledge or practical experience of airway ultrasound (Table 2).

Table 2. Baseline characteristics of the medical students

\begin{tabular}{lc}
\hline Characteristics & Value \\
\hline Age $(\mathrm{yr})$ & $27.6 \pm 3.2$ \\
Sex & \\
Male & $36(69)$ \\
Female & $16(31)$ \\
Previous knowledge of airway ultrasound & \\
Yes & $0(0)$ \\
No & $52(100)$ \\
\hline
\end{tabular}

Values are presented as mean \pm standard deviation or number (\%).

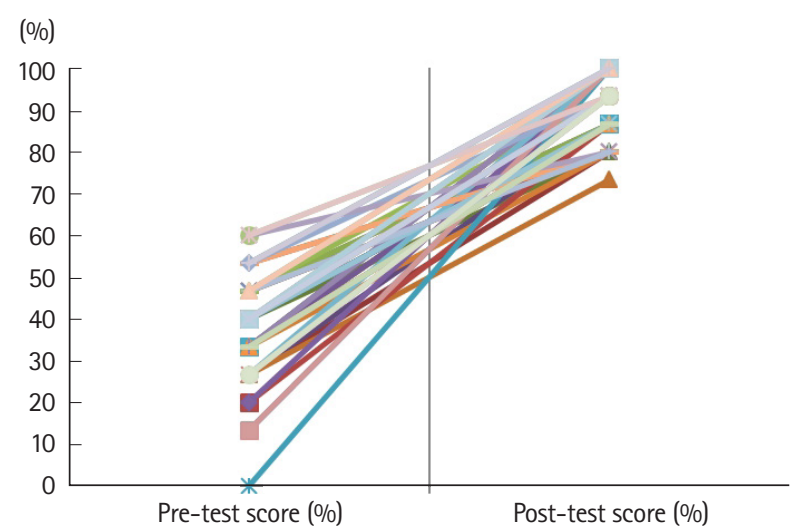

Fig. 2. Comparison of pretest and posttest scores. Improvement was visible in the medical students' post-educational cognitive skills. The mean score in the pretest was 38.7 and that in the posttest was 91.2.

\section{Cognitive skills}

Theoretical knowledge about POCUS-A significantly improved after training (38.7 $\pm 12.4 \%$ vs. $91.2 \pm 7.7 \%, P<0.001)$ (Fig. 2).

\section{Construct validity}

There was no statistically significant difference between the students' post-educational scores in theoretical knowledge and the scores of EM residents $(91.2 \pm 7.7 \%$ vs. $90.8 \pm 4.6 \%, P=0.551)$.

\section{Psychomotor skills}

Concerning the evaluation of ultrasound performance, the overall success rate of POCUS-A scan was 95.8\%. The success rate of each segment of the airway scan was 100\% for transverse scan of thyroid cartilage and cricoid cartilage, 96.2\% for transverse scan of trachea and esophagus below cricoid cartilage, 92.3\% for transverse scan of trachea and esophagus above sternal notch, 92.3\% for longitudinal scan of CTM, and 98.1\% for longitudinal scan of tracheal cartilage (Fig. 3).

\section{Posttest questionnaires}

In the post-training questionnaire, the students showed a high degree of satisfaction and understanding of POCUS-A education and high level of confidence in the practice of airway ultrasound in real patients in the future. Furthermore, students answered that the need for POCUS-A education was high in the medical school curriculum (Table 3).

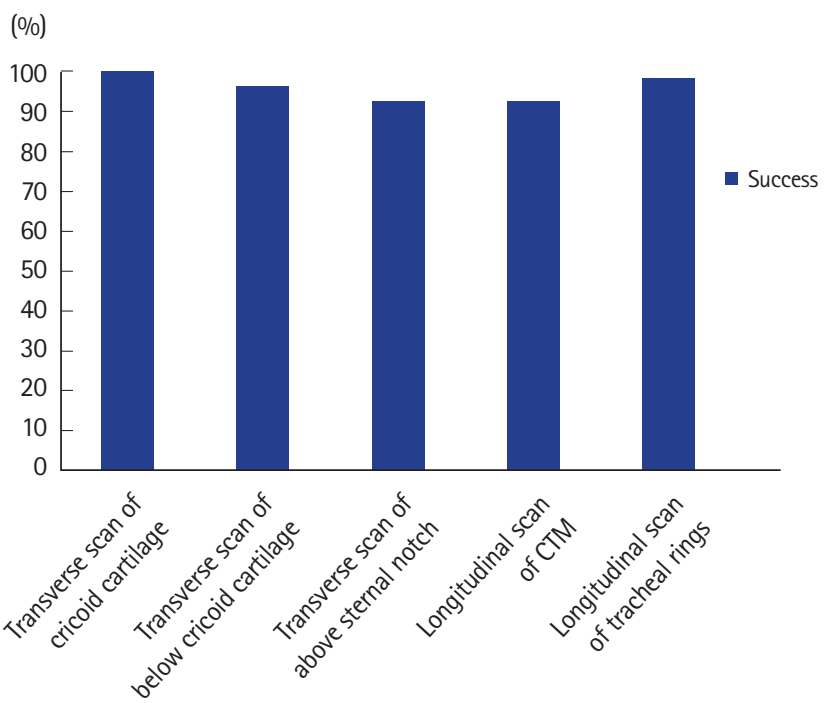

Fig. 3. Success rate of the airway sonography performance test. The success rates of transverse scans below cricoid cartilage and above sternal notch were $96.2 \%$ and $92.3 \%$, respectively, while the success rates for longitudinal scans of cricothyroid membrane (CTM) and tracheal rings were $92.3 \%$ and $98.1 \%$, respectively. 
Table 3. Students' responses to the post-training survey on POCUS-A education

\begin{tabular}{|c|c|c|c|c|c|}
\hline Likert scale $\mathrm{e}^{\mathrm{a})}$ & 1 & 2 & 3 & 4 & 5 \\
\hline Understanding of POCUS-A training & $0(0)$ & $0(0)$ & $12(23.1)$ & $19(36.5)$ & $21(40.4)$ \\
\hline Satisfaction with POCUS-A training & $0(0)$ & $0(0)$ & $0(0)$ & $23(44.2)$ & $29(55.8)$ \\
\hline Need for POCUS-A training within the medical curriculum & $0(0)$ & $0(0)$ & $5(9.6)$ & $27(51.9)$ & $20(38.5)$ \\
\hline Confidence and ability to perform POCUS-A in patients & $0(0)$ & $6(11.5)$ & 10 (19.2) & $27(51.9)$ & $9(17.3)$ \\
\hline
\end{tabular}

Values are presented as number (\%).

POCUS-A, point-of-care ultrasound of the airway.

${ }^{\text {a) }}$ Likert scale: $1=$ very low, $2=$ low, $3=$ moderate, $4=$ high, $5=$ very high.

\section{DISCUSSION}

The utility of airway ultrasonography has already been reported several times. Using airway ultrasonography, it is possible to monitor the accurate performance of endotracheal intubation in real time. In addition, in patients for whom the thyroid cartilage cannot be palpated, ultrasound can confirm the position of the CTM to facilitate cricothyroidotomy. ${ }^{9-13}$ Chou et al. ${ }^{7}$ evaluated the adequacy of endotracheal intubation in 112 patients who underwent tracheal intubation in an ED and confirmed its diagnostic accuracy using quantitative waveform capnography. They reported that the diagnostic accuracy was $98.2 \%$, sensitivity was 98.9\%, and specificity was 94.1\%. Especially intra-esophageal intubation can be anticipated by the presence of a double tract sign with a false second airway at the esophagus very specifically. ${ }^{14}$ Sustic et al. ${ }^{10}$ reported a case of tracheostomy using ultrasound in a morbidly obese patient. Siddiqui et al. ${ }^{15}$ reported that the use of ultrasound guidance for cricothyroidotomy in cadavers with poorly defined neck anatomy, with impalpable anatomical landmarks, increased the success rate of cricothyroidotomy 5.6fold and reduced the complications of surgery. Kristensen et al. ${ }^{16}$ reported the success rate of locating the CTM in an obese female volunteer with a body mass index of 45 by 35 anesthesiologists with 6 years of experience. The success rate of the conventional method was $37 \%$, and with ultrasound location it was $83 \%$. Thus, POCUS-A is very useful in airway management. However, education on it is not universal, and therefore, it is rarely used clinically.

For developing this educational program and evaluation methods, we assessed content validity by involving six specialists in POCUS-A and determined CVI, face validity, and construct validity with eight EM residents for improving the clarity of the educational items. Our prospective pilot study demonstrates that POCUS-A training for undergraduate students in medical schools facilitated successful ultrasound scanning and adequate interpretation of the test results. The students' self-assessed technical knowledge and confidence in the use of POCUS-A was enhanced, and their motivation to perform ultrasound was improved by their enjoyment of diagnosis through ultrasound. Although many studies reported successful education of medical students about target ultrasound, this study is the first to report on the effectiveness of teaching airway ultrasound.

Through this curriculum, students were able to improve both cognitive and psychomotor skills. Particularly in terms of knowledge evaluation, all the students answered the six questions by correctly distinguishing tracheal intubation from esophageal intubation. In this study, transverse scanning to detect esophageal intubation was performed in two different probe locations. Students showed a success rate of $92.3 \%$ in the location above the sternal notch proposed by Chou et al., ${ }^{7}$ but below the cricoid cartilage level, the success rate was higher at 96.2\%. Although there was no statistically significant difference, scanning at the lower cricoid cartilage level may help beginners to easily scan the trachea and esophagus simultaneously. Kristensen et al. ${ }^{9}$ reported the total success of ultrasonographic identification of the CTM performed by airway experts, but in this study, CTM scanning succeeded only in $92.3 \%$ of the students. The difficulty of anchoring the probe in the midline due to the protrusions of the thyroid cartilage might be the cause of failure. Many students complained that the probe was floating and it was difficult to attach it to necks during the scan of thyroid cartilage. Therefore, it is necessary to explain in detail that a vertical scan of the thyroid cartilage should begin at the inferior end of the protrusion in order to keep the probe attached.

Recently, POCUS examinations have become popular, and studies report the need for POCUS education of students. ${ }^{17-22}$ However, which type of POCUS programs students should be educated in has not been clarified. We recommend teaching students POCUS-A for the following reasons. First, POCUS-A can be used to instantly determine whether the life-sustaining airway is maintained appropriately, and facilitate identification of the CTM in difficult patients. Second, POCUS-A is associated with a very high success rate because it can be used to examine superficial structures compared to other target ultrasound interventions. Gogal- 
niceanu et al. ${ }^{17}$ reported $86.0 \%$ success rate with an education involving Focused Assessment for Sonography in Trauma in a group of 25 medical college students. Jeppesen and Bahner ${ }^{22}$ trained medical students in the Trinity protocol (hypotensive ultrasound protocol) and reported a $36 \%$ success rate. Thus, this study suggests that POCUS-A education can stimulate students' interest due to the much higher success rate than other target ultrasound education programs suggested above.

There are some limitations to this study. First, it is difficult to generalize the results due to the small sample size of 52 students, from a single medical college. Second, the students did not scan abnormal findings because they conducted ultrasonography on a normal standard patient. However, the purpose of this training was to observe the airway and esophagus simultaneously with ultrasound to confirm accurate intubation, and determine the position and depth of the CTM, which were fully observed in a standard patient. Further, the sonographic findings of esophageal intubation were available as image data for the students to easily distinguish and enhance their cognitive knowledge. Third, because this study was conducted only during their EM rotation, it did not evaluate the degree of knowledge retention in students after a specific period of time. Finally, ultrasonography was performed on healthy standard patients while lying comfortably, which was easier for students to obtain images than in a real-life clinical setting.

In summary, this study shows that a simple airway ultrasound education can improve the knowledge and image acquisition skills and interpretation ability of medical students. We believe that POCUS-A training should be considered when developing undergraduate ultrasonography education. Future studies will need to supplement this evaluation after a certain period of time in order to assess the retention of knowledge.

\section{CONFLICT OF INTEREST}

No potential conflict of interest relevant to this article was reported.

\section{SUPPLEMENTARY MATERIAL}

Supplementary Materials are available from: https://doi.org/10.15441/ ceem.18.061.

\section{REFERENCES}

1. Amini $R$, Stolz LA, Gross $A$, et al. Theme-based teaching of point-of-care ultrasound in undergraduate medical educa- tion. Intern Emerg Med 2015;10:613-8.

2. Soucy ZP, Mills LD. American academy of emergency medicine position statement: ultrasound should be integrated into undergraduate medical education curriculum. J Emerg Med 2015;49:89-90.

3. Mouratev G, Howe D, Hoppmann R, et al. Teaching medical students ultrasound to measure liver size: comparison with experienced clinicians using physical examination alone. Teach Learn Med 2013;25:84-8.

4. Osman A, Sum KM. Role of upper airway ultrasound in airway management. J Intensive Care 2016;4:52.

5. Garg R, Gupta A. Ultrasound: a promising tool for contemporary airway management. World J Clin Cases 2015;3:926-9.

6. Votruba J, Zemanova P, Lambert L, Vesela MM. The role of airway and endobronchial ultrasound in perioperative medicine. Biomed Res Int 2015;2015:754626.

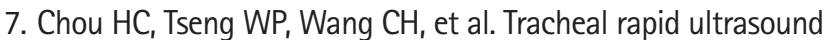
exam (T.R.U.E.) for confirming endotracheal tube placement during emergency intubation. Resuscitation 2011;82:127984.

8. Mallin $M$, Curtis $K$, Dawson $M$, Ockerse $P$, Ahern M. Accuracy of ultrasound-guided marking of the cricothyroid membrane before simulated failed intubation. Am J Emerg Med 2014;32: 61-3.

9. Kristensen MS, Teoh WH, Rudolph SS. Ultrasonographic identification of the cricothyroid membrane: best evidence, techniques, and clinical impact. Br J Anaesth 2016;117 Suppl 1:i3948.

10. Sustic A, Zupan Z, Antoncic I. Ultrasound-guided percutaneous dilatational tracheostomy with laryngeal mask airway control in a morbidly obese patient. J Clin Anesth 2004;16:121-3.

11. Ezri T, Gewurtz G, Sessler DI, et al. Prediction of difficult laryngoscopy in obese patients by ultrasound quantification of anterior neck soft tissue. Anaesthesia 2003;58:1111-4.

12. Wu J, Dong J, Ding Y, Zheng J. Role of anterior neck soft tissue quantifications by ultrasound in predicting difficult laryngoscopy. Med Sci Monit 2014;20:2343-50.

13. Pinto J, Cordeiro L, Pereira C, Gama R, Fernandes HL, Assuncao $\mathrm{J}$. Predicting difficult laryngoscopy using ultrasound measurement of distance from skin to epiglottis. J Crit Care 2016; 33:26-31.

14. Chou EH, Dickman E, Tsou PY, et al. Ultrasonography for confirmation of endotracheal tube placement: a systematic review and meta-analysis. Resuscitation 2015;90:97-103.

15. Siddiqui N, Arzola C, Friedman Z, Guerina L, You-Ten KE. UItrasound improves cricothyrotomy success in cadavers with poorly defined neck anatomy: a randomized control trial. An- 
esthesiology 2015;123:1033-41.

16. Kristensen MS, Teoh WH, Rudolph SS, et al. Structured approach to ultrasound-guided identification of the cricothyroid membrane: a randomized comparison with the palpation method in the morbidly obese. Br J Anaesth 2015;114:1003-4.

17. Gogalniceanu P, Sheena Y, Kashef E, Purkayastha S, Darzi A, Paraskeva $P$. Is basic emergency ultrasound training feasible as part of standard undergraduate medical education? J Surg Educ 2010;67:152-6.

18. Hoppmann RA, Rao W, Bell $F$, et al. The evolution of an integrated ultrasound curriculum (iUSC) for medical students: 9-year experience. Crit Ultrasound J 2015;7:18.

19. Hoppmann RA, Rao W, Poston MB, et al. An integrated ultra- sound curriculum (iUSC) for medical students: 4-year experience. Crit Ultrasound J 2011;3:1-12.

20. Rao S, van Holsbeeck L, Musial JL, et al. A pilot study of comprehensive ultrasound education at the Wayne State University School of Medicine: a pioneer year review. J Ultrasound Med 2008;27:745-9.

21. Griksaitis MJ, Scott MP, Finn GM. Twelve tips for teaching with ultrasound in the undergraduate curriculum. Med Teach 2014;36:19-24.

22. Jeppesen KM, Bahner DP. Teaching bedside sonography using peer mentoring: a prospective randomized trial. J Ultrasound Med 2012;31:455-9. 